\title{
O IMPACTO DA ATIVIDADE FÍSICA NA ARTRITE REUMATOIDE
}

\author{
Susana Santos Sampaio Lacerda Silva Escola Bahiana de Medicina e Saúde \\ Pública. \\ Selena Márcia Dubois Mendes Escola Bahiana de Medicina e Saúde \\ Pública. \\ Silvana Almeida Nascimento Ribas Escola Bahiana de Medicina e Saúde \\ Pública.
Abrahão Fontes Baptista Universidade Federal da Bahia. Pública. \\ Mittermayer Barreto Santiago Escola Bahiana de Medicina e Saúde \\ Kátia Nunes Sá Escola Bahiana de Medicina e Saúde \\ Pública.
}

Endereço para correspondência: sssilva1@sou.bahiana.edu.br

\begin{abstract}
Resumo
Objetivos: Verificar o impacto da atividade física na artrite reumatoide (AR) e a associação entre o nível de atividade física (NAF) com o nível de atividade da doença (NAD). Metodologia: Estudo observacional de corte transversal realizado em indivíduos com AR de um ambulatório de referência de Salvador-BA. Foram incluídos pacientes de ambos os sexos, com idade entre 18 e 65 anos, com diagnóstico médico confirmado para AR pelos critérios do Colégio Americano de Reumatologia; e excluídos pacientes com déficits cognitivos e doenças associadas que pudessem afetar o NAF. Na coleta de dados utilizou-se o International Physical Activity Questionnaire (IPAQ), um questionário sociodemográfico e foram aferidos peso e altura pela balança de precisão da marca Welmy. A coleta foi realizada nos dias em que os pacientes já eram assistidos. As variáveis independentes foram o NAF, idade e tempo de doença. A variável dependente foi o NAD. Para análise das variáveis categóricas NAF e NAD foi usado o Qui-quadrado e para análises numéricas idade e tempo de doença o ANOVA. Resultados: Não foi encontrada relação significativa entre o NAF e o NAD ( $\mathrm{p}=0,73)$, porém, entre a escolaridade e o NAF houve significância $(\mathrm{p}=0,06)$. Conclusão: Conclui-se que não houve associação entre o NAF e o NAD. A escolaridade demonstrou significância estatística nesta população, o que pode estar relacionado com acesso à informação da população estudada e o autocuidado.
\end{abstract}

Palavras-chave: Função; Atividade Física; Artrite Reumatoide.

\section{THE IMPACT OF PHYSICAL ACTIVITY IN RHEUMATOID ARTHRITIS}

\begin{abstract}
Objectives: To evaluate the impact of physical activity in RA and the association between physical activity level with Level of Disease Activity. Methodology: A cross-sectional observational study were conducted on individuals with RA form a referral center in Salvador - Bahia. Included are patients of both sexes, aged between 18 and 65 years, with a diagnosis confirmed for RA according American Rheumatology Association, and excluded patients with cognitive deficits and associated diseases that could affect the physical activity level. Data collection used the International Physical Activity Questionnaire (IPAQ), a demographic questionnaire, and measured weight and height for precision balance brand Welmy. Data collection was conducted in a referral outpatient Salvador on days when patients were already assisted. The independent variables were the physical activity level, age and disease duration. The dependent variable was the Level of Disease Activity. For analysis of categorical variables Level of Disease Activity and physical activity level was used Qhi-square analyzes and numerical age and disease duration ANOVA. Results: No significant relationship was found between the physical activity level and Level of Disease Activity $(p=0.73)$, however, between schooling and the physical activity level was significant $(\mathrm{p}=0.06)$. Conclusion: It was concluded that there was no association between
\end{abstract}


physical activity level and Level of Disease Activity. Schooling demonstrated statistical significance in this population, which may be related to access to information and self-care population.

Keywords: Function; Physical Activity; Rheumatoid Arthritis.

\section{INTRODUÇÃO}

A prevalência da Artrite Reumatoide (AR) é estimada em 0,5\% a 1,0\% da população adulta e as mulheres são duas a três vezes mais acometidas que os homens, sendo que o início geralmente ocorre entre os 30 e 50 anos. ${ }^{(1)}$ Fernandes et al relataram que $20 \%$ a $30 \%$ dos pacientes não tratados tornam-se incapacitados para o trabalho em dois a três anos do diagnóstico da doença. A AR é considerada uma doença inflamatória crônica, de origem autoimune, mas de etiologia ainda desconhecida, que causa dano progressivo no sistema musculoesquelético. ${ }^{(2)}$

O quadro clínico da doença é caracterizado por rigidez matinal com duração de ao menos uma hora, fadiga, mal-estar, edema de tecidos moles em áreas articulares, edemas simétricos, nódulos reumatoides, presença de fator reumatoide diagnosticado por exame clínico, erosões radiográficas e/ou osteopenia periarticular em articulações da mão e punho. ${ }^{(3)}$

Os sintomas de natureza musculoesquelética determinam diminuição da atividade física por estes pacientes, sendo o sedentarismo e a inatividade física observados freqüentemente nos pacientes com $\mathrm{AR} .{ }^{(4)} \mathrm{A}$ atividade física se refere a qualquer movimento que o corpo realize em função de contração muscular, com gasto energético acima do repouso $^{(5)}$ e é classificada segundo o International Physical Activity Questionnaire (IPAQ) ${ }^{(6)}$ em: sedentário, ativo, muito ativo, irregularmente ativo tipo A e irregularmente ativo tipo B. ${ }^{(7)}$

Os estudos revelam que a prática de atividade física é benéfica para o tratamento da AR. ${ }^{(8,9,10,11)}$ A prática de atividade física regular pode contribuir com o tratamento da AR no controle da atividade inflamatória, reduzindo a carga física causada pela doença, proporcionando não apenas melhora no quadro específico mas também redução da possibilidade do desenvolvimento de co-morbidades que podem agravar a funcionalidade do indivíduo. $^{(3)}$

Por ser uma doença com flutuação entre exacerbação de sintomas e períodos de remissão, tem sido recomendado que indivíduos, tanto em estudos de delineamento de perfil como em respostas a tratamentos, devem ser categorizados segundo o Nível de Atividade da Doença (NAD) que apresenta quatro estratos: período de remissão, NAD baixa, NAD 
moderada e NAD alta. Esta estratificação é realizada a partir de exames laboratoriais e sintomatologia dolorosa e sobre presença de edemas nas articulações através do Disease Activite Score - DAS-28. ${ }^{(1)}$

Apesar das evidências da melhora do quadro geral do paciente com AR com a inclusão da atividade física no seu programa de tratamento não há estudo que relacione o nível de atividade física (NAF) com o NAD da AR. Os objetivos deste estudo são verificar o impacto da atividade física na AR e verificar a associação entre o NAF com o NAD.

\section{METODOLOGIA}

Trata-se de um estudo observacional de corte transversal realizado no Ambulatório de Reumatologia do Ambulatório Docente Assistencial da Bahiana (ADAB), da Escola Bahiana de Medicina e Saúde Pública (EBMSP), referência em reumatologia em Salvador-BA. Foram incluídos no trabalho pacientes com AR de ambos os sexos, com idade de 18 a 65 anos, com diagnóstico médico confirmado de AR, e excluídos aqueles com déficit cognitivo e com outras doenças crônicas, degenerativas, neurológicas, ortopédicas, pneumológicas e cardiológicas associadas que possam afetar o nível de atividade física.

O cálculo amostral foi realizado através da calculadora do Laboratório de Epidemiologia e Estatística da USP (LEE), ${ }^{(12)}$ sendo necessária uma amostra de 71 pacientes portadores de AR para avaliar o nível de atividade física. Foi considerado um desvio padrão de 30 e diferença a ser detectada de 10 de nível de atividade avaliada pelo IPAQ para um nível de significância de $5 \%$ e poder de teste de $80 \%$ em hipótese bicaudal.

O Comitê de Ética em Pesquisa da EBMSP, após a análise do ponto de vista bioético do Protocolo $n^{\circ}$ 002/2011 aprovou a realização da pesquisa. Cada voluntário assinou um Termo de Consentimento Livre e Esclarecido (TCLE), conforme determina o decreto-lei 196/96 do CNS para a pesquisa em humanos que garantirá o anonimato destes.

Dos 97 pacientes cadastrados no serviço que compareceram à coleta, nove foram eliminados por apresentar idade superior a 65 anos, dois por dados incompletos sobre IMC, um por presença de CA de tireóide, três por insuficiência cardíaca, um por fibrose pulmonar, um por labirintite, um por depressão, um por espondiloartrose lombar e três por lúpus, e quatro possuíam mais de quatro doenças associadas.

A coleta dos dados foi realizada entre os meses de fevereiro e agosto de 2012, no referido ambulatório, nos dias de atendimento, em ambiente privativo. Os pacientes eram 
contatados por telefone e instruídos a comparecer em jejum ao local da pesquisa. Foram esclarecidos das técnicas aplicadas e dos objetivos da pesquisa. Depois os participantes eram dirigidos ao local da coleta de sangue e radiografia de punho e mãos. Foi aplicado um Questionário Sociodemográfico (Anexo B) e o IPAQ (Anexo A), além de aferidos peso e altura através da balança de precisão da marca Welmy.

O IPAQ ${ }^{(6)}$ procura determinar o nível de atividade física em cinco seções: trabalho, transporte, casa e atenção à família, lazer e tempo sentado, subdivididas em atividades moderadas e vigorosas. Pergunta-se sobre a duração e a frequência dessas atividades e pontuação final é expressa pela somatória do tempo e frequência de atividade física em minutos, na semana anterior ao estudo, em cada seção do questionário. ${ }^{(7)}$ São classificados como muito ativos indivíduos que cumpriram $\geq 5$ dias por semana, sendo $\geq 30$ minutos por sessão de atividade vigorosa, ou $\geq 3$ dias por semana e $\geq 20$ minutos por sessão de atividade vigorosa somados a atividade moderada e/ou caminhada por $\geq 5$ dias por semana, sendo $\geq 30$ minutos por sessão. Indivíduos considerados ativos cumpriram $\geq 3$ dias por semana e $\geq 20$ minutos por sessão de atividade vigorosa ou atividade moderada ou caminhada por $\geq 5$ dias por semana e $\geq 30$ minutos por sessão, ou qualquer atividade somada (caminhada, atividade vigorosa ou moderada) por $\geq 5$ dias por semana e $\geq 150$ minutos por semana. Indivíduos irregularmente ativos A foram aqueles que atingiram pelo menos um dos critérios da recomendação quanto à frequência ou quanto à duração da atividade física em frequência de 5 dias por semana ou duração de 150 minutos por semana. Os irregularmente ativos B foram os que não atingiram nenhum dos critérios quanto à frequência nem quanto à duração. Os sedentários não realizaram nenhuma atividade física por pelo menos 10 minutos contínuos durante a semana.

O NAD foi definido de acordo com valores da Velocidade da Hemossedimentação (VHS), da Escala Visual Analógica de Dor (EVA-D) e do número de articulações edemaciadas e número de articulações dolorosas (Disease Activite Score - DAS 28). O índice de remissão é definido pelo ponto de corte em $\leq 2,4$; o índice de baixa atividade caracterizado por 2,5 a 3,6; o índice de atividade moderada com o valor de 3,7 a 5,5 e o índice de alta atividade da doença se caracteriza pelo valor $\geq 5,6 .^{(13)}$

O pacote estatístico para a tabulação e análise dos dados será o Statistical Package for Social Science (SPSS), versão 14.0. As variáveis independentes do estudo são o NAF, o tempo da doença e a idade, e a variável dependente é o NAD. Para descrever o tempo da doença e a idade serão utilizados a média e o desvio padrão e para descrever grau da AR e 
nível de atividade física serão utilizadas as proporções. Para verificar a associação entre as variáveis serão utilizados o Qui-quadrado $\left(\mathrm{X}^{2}\right)$ e ANOVA. Para todas as análises foi considerado um nível de significância de 5\% $(\alpha=0,05)$.

\section{RESULTADOS}

A amostra foi composta por 71 indivíduos, sendo 64 do sexo feminino $(90,1 \%)$ e sete do sexo masculino (9,9\%), com idade média de 50,28 e desvio padrão de 10,2 (Tabela 1). A maioria desses indivíduos refere cor da pele parda $(45,1 \%)$ e pertence à classe $C(69,1 \%)$. A maioria da amostra não possui ensino superior completo $(35,2 \%)$ e trabalha na área de prestação de serviços $(53,5 \%)$ (Tabela 1$)$. 
Tabela 1 - Dados sociodemográficos de 71 pacientes com Artrite Reumatoide. Salvador-BA

\begin{tabular}{|c|c|}
\hline Variável & Média $\pm D P$ ou n $(\%)$ \\
\hline Idade (anos) & $50,28 / \pm 10,2$ \\
\hline Sexo Feminino & $64(90,1 \%)$ \\
\hline \multicolumn{2}{|l|}{ Ocupação } \\
\hline Aposentado & $7(9,9 \%)$ \\
\hline Ocupações administrativas & $7(9,9 \%)$ \\
\hline Ocupações técnicas, científicas, artísticas e afins & $6(8,4 \%)$ \\
\hline $\begin{array}{l}\text { Ocupações das indústrias de transformação e } \\
\text { construção civil }\end{array}$ & $3(4,2 \%)$ \\
\hline Ocupações do comércio e atividades auxiliares & $3(4,2 \%)$ \\
\hline Ocupações da prestação de serviços & $38(53,5 \%)$ \\
\hline Sem atividade definida & $7(9,9 \%)$ \\
\hline \multicolumn{2}{|l|}{ Cor da Pele } \\
\hline Branca & $5(7 \%)$ \\
\hline Preta & $31(43,7 \%)$ \\
\hline Vermelha & $3(4,2 \%)$ \\
\hline Pardo & $32(45,1 \%)$ \\
\hline \multicolumn{2}{|l|}{ Escolaridade } \\
\hline Analfabeto/Primário Incompleto & $8(11,3 \%)$ \\
\hline Primário Completo/Ginásio Incompleto & $16(22,5 \%)$ \\
\hline Ginásio Completo/Colegial Incompleto & $16(22,5 \%)$ \\
\hline Colegial Completo/Superior Incompleto & $25(35,2 \%)$ \\
\hline Superior Completo & $6(8,5 \%)$ \\
\hline \multicolumn{2}{|l|}{ Classe Social } \\
\hline A1 & $1(1,4 \%)$ \\
\hline $\mathrm{A} 2$ & $1(1,4 \%)$ \\
\hline B1 & $2(2,8 \%)$ \\
\hline B2 & $12(16,9 \%)$ \\
\hline $\mathrm{C} 1$ & $18(25,4 \%)$ \\
\hline $\mathrm{C} 2$ & $31(43,7 \%)$ \\
\hline $\mathrm{D}$ & $6(8,5 \%)$ \\
\hline
\end{tabular}

$\mathrm{DP}=$ desvio padrão; $\mathrm{n}(\%)=$ número de pacientes 
O IMC médio da população foi 27,9 com desvio padrão de 5,59, o que caracteriza sobrepeso. O tempo de doença tem média de 12,27 anos com desvio padrão de 7,12, sendo que a maioria fez fisioterapia anteriormente $(74,6 \%)$, mas não faz atualmente $(83,1 \%)$.

Quanto às doenças associadas, $29(40,9 \%)$ não possuem co-morbidades. A doença mais comum associada à AR foi a hipertensão arterial sistêmica (38\%).

A fim de estabelecer a prevalência do nível de atividade física segundo o IPAQ, dos 71 entrevistados, foram classificadas 56 pessoas $(78,9 \%)$ como ativas. Nenhum indivíduo da amostra estudada foi classificado como sedentário.

Quanto ao nível de atividade da doença, apenas uma pessoa $(1,4 \%)$ foi classificada como baixa atividade e $44(62 \%)$ como tendo uma alta atividade da doença. Nenhum indivíduo apresentou-se em período de remissão (Tabela 2).

Não houve associação entre o nível de atividade física e o nível de atividade da doença (Tabela 3).

Foi encontrada associação entre nível de atividade física com a escolaridade (Tabela 4), porém, sexo, cor da pele, classe social, se faz uso de medicamento, se faz ou fazia fisioterapia ou terapia ocupacional, tipo de ocupação, tabagismo ou uso de álcool não foram significativos em relação à atividade física. Utilizando o teste ANOVA não houve associação entre o nível de atividade física com idade, tempo de doença, IMC e nível de atividade da doença. 
Tabela 2 - Dados clínicos de 71 pacientes com Artrite Reumatoide, Salvador-BA

\begin{tabular}{ll}
\hline Variável & $\begin{array}{l}\text { Média } \pm \text { DP ou } \\
\mathbf{n}(\%)\end{array}$ \\
\hline Tempo de doença & $12,27 \pm 7,12$ \\
IMC & $27,90 \pm 5,59$
\end{tabular}

Fisioterapia Passada

Sim $\quad 53(74,6 \%)$

Fisioterapia Atual

Não $59(83,1 \%)$

Terapia Ocupacional

Não $\quad 66(93 \%)$

Medicamentos

Sim $\quad 69(97,2 \%)$

Fumo

$\begin{array}{ll}\text { Não } & 60(84,5 \%) \\ \text { Sim } & 4(5,6 \%) \\ \text { Ex-Fumante } & 7(9,9 \%)\end{array}$

Álcool

Não $\quad 56(78,9 \%)$

\section{Nível de Atividade Física}

$\begin{array}{ll}\text { Ativo } & 56(78,9 \%) \\ \text { Irregularmente Ativo A } & 9(12,7 \%) \\ \text { Irregularmente Ativo B } & 2(2,8 \%) \\ \text { Muito Ativo } & 4(5,6 \%)\end{array}$

Doenças Associadas

Não $29(40,8 \%)$

Hipertensão Arterial Sistêmica 27 (38\%)

Diabetes Melitus $\quad 5(7 \%)$

Doenças Hepáticas $2(2,8 \%)$

Doenças Muscoloesqueléticas $\quad 5(7 \%)$

Doenças Oculares $\quad 3(4,2 \%)$

Outras $\quad 11(15,5 \%)$

$\mathrm{DP}=$ desvio padrão; n(\%) número de pacientes 
Tabela 3 - Associação entre o nível de atividade física com o nível de atividade da doença

\section{Nível de atividade física}

Ativo Irregularmente Ativo A Irregularmente Ativo B Muito Ativo Total

\begin{tabular}{lccccc}
\hline $\begin{array}{l}\text { Nível de atividade } \\
\text { da doença }\end{array}$ & & & & & \\
Baixa atividade & 1 & 0 & 0 & 0 & 1 \\
Moderada atividade & 22 & 1 & 1 & 2 & 26 \\
Alta atividade & 33 & 8 & 1 & 2 & 44 \\
Total & 56 & 9 & 2 & 4 & 71 \\
\hline
\end{tabular}

Qui-quadrado, $\mathrm{p}=0,73$

Tabela 4 - Associação entre o nível de atividade física e a escolaridade

\begin{tabular}{|c|c|c|c|c|c|}
\hline \multirow[b]{2}{*}{ Escolaridade } & \multicolumn{5}{|c|}{ Nível de atividade física } \\
\hline & Ativo & $\begin{array}{l}\text { Irregularmente ativo } \\
\mathrm{A}\end{array}$ & $\begin{array}{c}\text { Irregularmente ativo } \\
\mathrm{B}\end{array}$ & $\begin{array}{l}\text { Muito } \\
\text { ativo }\end{array}$ & Total \\
\hline Analfabeto/Primário incompleto & 6 & 2 & 0 & 0 & 8 \\
\hline $\begin{array}{l}\text { Primário completo/Ginásio } \\
\text { incompleto }\end{array}$ & 10 & 3 & 0 & 3 & 16 \\
\hline $\begin{array}{l}\text { Ginásio completo/Colegial } \\
\text { incompleto }\end{array}$ & 11 & 3 & 2 & 0 & 16 \\
\hline $\begin{array}{l}\text { Colegial Completo/Superior } \\
\text { incompleto }\end{array}$ & 24 & 0 & 0 & 1 & 25 \\
\hline Superior completo & 5 & 1 & 0 & 0 & 6 \\
\hline Total & 56 & 9 & 2 & 4 & 71 \\
\hline
\end{tabular}

*ANOVA Oneway, p=0,06

\section{DISCUSSÃO}

Este estudo buscou verificar a associação entre o nível de atividade física e o nível de atividade da doença, não encontrando associação entre estes fatores, embora tenha sido observado que o nível de escolaridade apresentou associação com a prática regular de atividade da vida diária que requerem esforço físico. Foi verificada uma dificuldade na comparação dos achados deste estudo com os dados disponíveis na literatura sobre o tema devido à falta de padronização no uso de instrumentos de coleta para avaliar nível de atividade física.

A maioria dos autores não utiliza o IPAQ como uma forma de categorizar o nível de atividade física segundo o consenso proposto pelo Celafisc, ${ }^{(7)}$ mas sim como uma forma de calcular a demanda energética ${ }^{(14,15)}$ ou a quantidade total de minutos gastos por dia em determinadas atividades sem considerar a frequência. ${ }^{(5,16)}$ Corroborando com os achados desta pesquisa, Figueredo ${ }^{(17)}$ avaliou a qualidade de vida e sua associação com a atividade física nos 
diferentes contextos da vida diária de pacientes com osteoartrite e encontrou na maioria dessa população piora dos aspectos físicos da qualidade de vida, mas com manutenção das atividades físicas cotidianas. Da sua amostra de 100 indivíduos estudados, $70 \%$ foram classificados como ativos e muito ativos.

Nossa amostra apresenta altos níveis de atividade da doença e maior prevalência em ocupações ligadas a prestação de serviços, que exigem um maior esforço físico do indivíduo. Esta carga física elevada em um indivíduo com uma doença de grande repercussão física como a AR leva a uma saída precoce do mercado de trabalho. ${ }^{(2)}$ Há estudos que relatam a alta prevalência de indivíduos com AR desempregados ou que realizam tarefas domésticas. ${ }^{(18,19)}$ Altas taxas de desemprego ou trabalhos de característica braçal podem estar ligadas a uma inserção em classes sociais menos elevadas. Assim como nesta pesquisa, há estudo que mostra indivíduos com AR sendo em sua maioria pertencentes a uma classe social baixa. ${ }^{(19)}$ Apesar disso, não foi encontrada significância estatística entre NAF e classe social.

A cor da pele com maior prevalência na população estudada varia entre outros estudos, ${ }^{(1,19,20)}$ mas os autores da pesquisa acreditam que este dado está ligado ao local onde os estudos foram feitos, já que a AR apresenta incidência parecida em todos os grupos étnicos. A média de tempo de doença não varia muito entre os estudos, ${ }^{(18-20)}$ o mesmo com a média de idade ${ }^{(18,20,21)}$ e IMC. ${ }^{(4,22)}$

Um estudo sobre a avaliação da atividade física e nutricional de pacientes com Lúpus Eritematoso Sistêmico ${ }^{(22)}$ também não mostrou significância estatística entre o NAF e o IMC. Diferente dessa pesquisa, a população da amostra foi em sua maioria classificada como insuficientemente ativa. O menor nível de atividade física desta população talvez esteja relacionado às doenças associadas presentes na população estudada, como nefrites, serosites, doenças neuropsiquiátricas, entre outros.

Vários estudos ${ }^{(1,4,19,20,23,24)}$ verificam que grande parte da população com AR também sofre com doenças extra articulares associadas, principalmente $\operatorname{HAS}^{(4,20,23)}$ e comprometimento ocular. ${ }^{(4,19)}$ Diferentes doenças podem ocorrer em conseqüência do uso prolongado de anti-inflamatórios e drogas modificadoras do curso da doença ou da própria característica comprometimento sistêmico da doença, que não se restringe a manifestações articulares, o que pode influenciar no NAF.

Relacionando-se com este estudo, níveis de escolaridade baixa foram encontrados em alguns estudos. ${ }^{(18,20)}$ Níveis de escolaridade mais baixos estão relacionados à maior incidência de fatores de risco para doenças crônicas, especialmente diabetes e as cardiovasculares, que 
são umas das principais doenças associadas com AR. ${ }^{(4)}$ Pode ser que o menor acesso à informação devido ao baixo nível de escolaridade e à menor preocupação com a saúde geral poderiam estar levando a associação encontrada entre escolaridade e AR. ${ }^{(25)}$

A homogeneidade da amostra possa ter interferido nos resultados do estudo por não abranger totalmente a população acometida pela AR. Pode ter havido viés na coleta de dados quanto às doenças associadas, já que estas foram relatadas pelos pacientes, sem exame que confirmassem a existência de outras co-morbidades. Por fim, o uso não padronizado do IPAQ pelos pesquisadores de forma geral dificulta uma análise comparativa entre os estudos.

Recomenda-se que em próximos estudos o tamanho da amostra seja aumentado para permitir análises mais precisas, assim como o uso padronizado do IPAQ para a categorização do nível de atividade física. Avaliar o nível de atividade física em pessoas com doenças crônicas é essencial para produzir evidências de sua recomendação em uma população que apresenta tantos impactos físicos, gera custos elevados para toda a sociedade e sofrimento humano. Caso seja comprovado que a prática regular de atividade física possa reduzir o nível de atividade da doença em estudos longitudinais, pode-se supor um impacto positivo na qualidade e expectativa de vida de uma população que faz uso regular de tantas drogas que apresentam efeito colateral importante.

Com base no conhecimento atual, é difícil definir o nível de atividade física da população acometida com AR pois ainda há uma discrepância entre os conceitos de atividade física e exercício físico na literatura. O nível de atividade física na população estudada ainda não permite dados conclusivos, embora os níveis elevados demonstrados neste estudo pareçam estar associados às ocupações em trabalhos domésticos e outras atividades braçais exercidas por uma população com baixo nível de escolaridade e classe social. Embora não tenha sido verificada uma associação entre nível de atividade física e nível de atividade da doença, a baixa escolaridade influenciou significativamente a prática regular de atividade física o que aponta para a multidimensionalidade dos problemas de saúde envolvidos com a artrite reumatoide.

\section{Agradecimentos}

Agradecemos à Escola Bahiana de Medicina e Saúde Pública que acolheu a pesquisa, a todos que fizeram parte da equipe que participou da coleta de dados para este trabalho, ao Grupo de Pesquisa em Dinâmica do Sistema Neuromuscoloesquelético. 


\section{REFERÊNCIAS}

1. Mota LM, Cruza BA, Brenol CV, Pereira IA, Rezende-Fronza LS, Bertolo MB et al. Diretrizes para diagnóstico da artrite reumatoide. Rev. bras. reumatol. [periódico online]. 2013; 53(2):141-157. Disponível em: http://www.scielo.br/scielo.php?pid=S0482$50042013000200003 \&$ script=sci_arttext

2. Fernandes EA, Castro Junior MR, Mitraud SAV, Kubota ES, Fernades ARC. Ultrasonografia na artrite reumatoide: aplicabilidade e perspectivas. Rev. bras. reumatol. 2008; 48(1):76-80.

3. Kulkamp W, Dario AB, Gevaerd MS, Domenech SC. Artrite reumatoide e exercício físico: resgate histórico e cenário atual. Rev. bras. ativ. fís. Saúde. 2009;14(1):55-64.

4. Brenol CV, Monticielo AO, Xavier RC, Brenol JCT. Artrite reumatoide e aterosclerose. AMB rev. Assoc. Med. Bras. 2007;53(5):105-106.

5. Matsudo VKR, Calmona CO. Osteoartrose e atividade física. Diagn. tratamento, 2009;14(4):146-151.

6. Celafisc - Centro Coordenador do Ipacs no Brasil. Questionário Internacional de Atividade Física IPAQ. 2013. [acesso em 2013 nov. 16]. Disponível em:

www.rc.unesp.br/ib/efisica/ekokubun/afsaude/IPAQVL.doc

7. Celafiscs - Centro Coordenador do Ipac no Brasil. Classificação de atividade física Ipac. 2007. [acesso em 2013 nov. 16]. Disponível em:

www.celafiscs.institucional.ws/65/questionarios.html

8. Bértolo MB, Brenol CV, Schainberg CG, Neubarth F, Lima FA, Laurindo IM et al. Atualização do consenso brasileiro no diagnóstico e tratamento da artrite reumatoide. Rev. bras. reumatol. 2007;47(3):151-9.

9. Santoni FC, Freitas SP, Oliveira J, Mesquita RA. Hidroterapia e qualidade de vida de um portador de artrite reumatoide juvenil. Fisioter mov. 2007;20(1):101-8.

10. Associação Médica Brasileira; Conselho Federal de Medicina. Artrite reumatoide: diagnóstico e tratamento. Projeto Diretrizes. 2002.

11. Ferreira LRF, Pestana PR, Oliveira J, Mesquita-Ferrari RA. Efeitos da reabilitação aquática na sintomatologia e qualidadede vida de portadoras de artrite reumatoide. Fisioter. pesqui. 2008;15(2):136-41.

12. Laboratório de Epidemiologia e Estatística da USP. 2013. [acesso em 2013 nov. 19]. Disponível em: http://www.lee.dante.br/pesquisa/amostragem/calculo_amostra.html

13. Pinheiro GRC. Instrumentos de medida da atividade da artrite reumatoide: porque e como empregá-los. Rev. bras. reumatol. 2007;47(5):362-365. 
14. Benedetti TRB, Antunes PC, Rodriguez-Añez CR, Mazo GZ, Petroski EL.

Reprodutibilidade e validade do Questionário Internacional de Atividade Física (IPAQ) em homens idosos. Rev. bras. med. esporte. 2007;13(1):11-6.

15. Alves MAS, Bueno FR, Haraguchi LIH, Correa FR, Dourado VZ. Correlação entre a média do número de passos diário e o teste de caminhada de seis minutos em adultos e idosos assintomáticos. Fisioter. Pesqui. 2013; 20(2):123-129.

16. Breda CA, Rodacki ALF, Leite N, Homann D, Goes SM, Stefanello JMF. Nível de atividade física e desempenho físico no teste de caminhada de 6 minutos em mulheres com fibromialgia. Rev. bras. reumatol. 2013;53(3):276-281.

17. Figueredo Neto EM, Queluz TT, Freire BFA. Atividade física e sua associação com qualidade de vida em pacientes com osteoartrite. Rev. bras. reumatol. 2011;51(6):539-544.

18. Corbacho ML. Avaliação da capacidade funcional e da qualidade de vida de pacientes com artrite reumatoide. Rev. bras. reumatol. 2010;50(1):31-43.

19. Louzada-Junior P, Souza BDB, Toledo RA, Ciconelli RM. Análise descritiva das características demográficas e clínicas de pacientes com artrite reumatoide no estado de São Paulo. Rev. bras. reumatol. 2007;47(1):84-90.

20. Moura MC, Zakszewski PTS, Silva MBG, Skare TL. Perfil dos pacientes com manifestações extra-articulares de artrite reumatoide de um serviço ambulatorial em Curitiba, Sul do Brasil. Rev. bras. reumatol. 2012; 52(5):686-694.

21. David JM, Mattei RA, Mauad JL, Almeida LD, Nogueira MA, Menolli PVS et al. Estudo clínico e laboratorial de pacientes com artrite reumatoide diagnosticados em serviços de reumatologia em Cascavel, PR, Brasil. Rev. bras. reumatol. 2013;53(1):61-65.

22. Santos FMM, Borges MC, Correia MITD, Telles RW, Lanna CCD. Avaliação do estado nutricional e da atividade física em pacientes com lúpus eritematoso sistêmico. Rev. bras. reumatol. 2010;50(6):631-45.

23. Pereira IA, da Mota LM, Cruz BA, Brenol CV, Rezende-Fronza LS, Bertolo MB et al. Consenso 2012 da Sociedade Brasileira de Reumatologia sobre o manejo de comorbidades em pacientes com o diagnostico de Artrite Reumatoide. Rev. bras. reumatol. 2012;52(4):474-95.

24. Pereira I. Artrite reumatoide: por que tratar apenas a artrite, sabendo que comorbidades são comuns e determinam morbidade e mortalidade Rev. bras. reumatol. 2012;52(4):472-473.

25. Iser BPM, Yokota RTC, Sá NNB, Moura L, Malta DC. Prevalência de fatores de risco e proteção para doenças crônicas nas capitais do Brasil - principais resultados do Vigitel 2010. Ciênc. saúde coletiva. 2012; 17(9):2343-2356. 